\title{
EMERGENCE OF ATTACHED RECIRCULATING EDDY FOR FLOW AROUND A CIRCULAR CYLINDER ASYMMETRICALLY PLACED IN A CHANNEL
}

\section{Tsung-Chan Hsieh}

Department of Systems Engineering and Naval Architecture, National Taiwan Ocean University, 2, Pei-Ning Road, Keelung, Taiwan.

Jiahn-Horng Chen

Department of Systems Engineering and Naval Architecture, National Taiwan Ocean University, 2, Pei-Ning Road, Keelung,Taiwan., b0105@mail.ntou.edu.tw

Follow this and additional works at: https://jmstt.ntou.edu.tw/journal

Part of the Engineering Commons

\section{Recommended Citation}

Hsieh, Tsung-Chan and Chen, Jiahn-Horng (2006) "EMERGENCE OF ATTACHED RECIRCULATING EDDY FOR FLOW AROUND A CIRCULAR CYLINDER ASYMMETRICALLY PLACED IN A CHANNEL," Journal of Marine Science and Technology. Vol. 14: Iss. 3, Article 3.

DOI: $10.51400 / 2709-6998.2068$

Available at: https://jmstt.ntou.edu.tw/journal/vol14/iss3/3

This Research Article is brought to you for free and open access by Journal of Marine Science and Technology. It has been accepted for inclusion in Journal of Marine Science and Technology by an authorized editor of Journal of Marine Science and Technology. 


\section{EMERGENCE OF ATTACHED RECIRCULATING EDDY FOR FLOW AROUND A}

CIRCULAR CYLINDER ASYMMETRICALLY PLACED IN A CHANNEL

\section{Acknowledgements}

This work was supported by the National Science Council, Republic of China, under grant NSC 93-2611E-019-021. The authors would like to express their thanks for this support. 


\title{
EMERGENCE OF ATTACHED RECIRCULATING EDDY FOR FLOW AROUND A CIRCULAR CYLINDER ASYMMETRICALLY PLACED IN A CHANNEL
}

\author{
Tsung-Chan Hsieh* and Jiahn-Horng Chen*
}

Key words: circular cylinder, asymmetry, recirculating flow, laminar separation, channel flow.

\section{ABSTRACT}

This study discusses the emergence and development of cylinder-attached recirculating regions appearing in a fully developed flow past a circular cylinder asymmetrically confined between two infinite parallel plates. We employed a finite-volume method with the SIMPLE algorithm to compute the flow fields for various sizes and asymmetrical settings of circular cylinders. Two geometrical parameters, the blockage and asymmetry coefficients, are varied in computations. A series of computations show that the flow evolves a single asymmetrical recirculating region for the Reynolds number beyond some critical value. Its development in length bears a linear relation to the Reynolds number. Its growth rate depends on the two geometrical parameters. In addition, the computations also show that as the cylinder is moved toward one of the plates, the critical Reynolds number becomes larger. It seems to imply that the existence of lateral plate delays the formation of recirculating region.

\section{INTRODUCTION}

The viscous flow phenomena behind a circular cylinder have been a classical problem in fluid mechanics. They have been vastly investigated theoretically, experimentally, and computationally, owing to its scientific and engineering significances. Numerous reports are available in literature. Recently, Zdravkovich [30, 31] has systematically compiled works of past several decades and attempted to establish a thorough reference.

Early studies were devoted mainly to the flow in an unbounded setting. The pioneering investigations are due to experimental work by Taneda [24], Tritton [28], Nishioka \& Sato [19], Coutanceau and Bouard [10, 11],

Paper Submitted 10/12/05, Accepted 02/09/06. Author for Correspondence: Jiahn-Horng Chen. E-mail: b0105@mail.ntou.edu.tw.

*Department of Systems Engineering and Naval Architecture, National Taiwan Ocean University, 2, Pei-Ning Road, Keelung, Taiwan. and Gerrard et al. [15]. Employing various experimental facilities, they attempted to capture the flow phenomena in an unbounded field. Their efforts lay the foundation to understanding this basic flow and their conclusions indicate that the flow separates from the cylinder at a Reynolds number about 4-6.

As computation techniques make rapid progress, numerical studies also make significant contributions to this problem. Many procedures have been specifically devised for some particular flow phenomena in an unbounded domain, e.g. Forngerg [13, 14], Anderson and Reider [4], Sohankar et al. [23], Lai and Peskin [17], and Tang et al. [26]. Among them, some focused on the treatment of boundary conditions in truncated domains and others developed new schemes for more accurate computations. Nevertheless, all experimental studies are not truly unbounded in that facilities are inevitably of finite sizes. Neither do computational studies because of finite domain truncations. Of course, early researchers also paid heed to this fact even though they attempted to realize the unbounded flow. They observed that the existence of walls had significant influence on the flow development. Grove et al. [16], Acrivos et al. [1], and Coutanceau and Bouard [10, 11] conducted a series of careful experiments to examine the effect of confining walls. Their conclusions show that the critical Reynolds number at which the flow begins to separate and the recirculating region forms increased as the blockage coefficient, defined as the ratio of the cylinder diameter to the characteristic length of the experimental facility, is increased. Furthermore, the effect due to confining walls is not negligible even at a small value of blockage coefficient, say 0.07. In computations, Behr et al. [6] studied the effect of lateral truncation on flow development. They concluded that "the minimum distance at which the truncation influence vanishes has been found to be larger than what is commonly assumed."

In fact, the existence of lateral confining walls not 
only affects the flow development but also introduces new physical phenomena not observable in an unbounded domain. These arise mainly due to interactions of the flow behind the cylinder with the shear effects from the walls. Therefore, many studies have recently been devoted to examining these interesting phenomena owing to the presence of a single wall in a plane boundary layer or two walls in a channel flow.

There have been some experimental reports available in the literature to discuss the flow past a circular cylinder in a plane boundary layer at moderately high Reynolds numbers (e.g., Bearman and Zdravkovich [5], Taniguchi and Miyakoshi [27], and Lei et al. [18]). The vortex shedding phenomenon and its flow pattern and force variation are of primary interest in these studies. Their observations indicate that as the cylinder is closer than some critical distance from the wall, the vortex shedding is suppressed.

As for the flow between two confining walls, e.g. channel flow, most studies focus on the cylinder placed symmetrically in the channel. Smith [22] employed the asymptotic theory to study the separating and reattaching flow past a bluff body. He showed theoretically that the confining walls could exert considerable influence on the eddy, even if they were widely spaced. Later, Chen et al. [9] ascertained how the steady flow past a circular cylinder lost stability as the Reynolds number was increased by confining it symmetrically between two parallel planes. They also studied the development of attached vortices including their length growth rates and critical Reynolds numbers for different blockage coefficients. In addition, Chen [8] revealed that the growth of the separation bubbles varied significantly for different boundary setups. More recently, Turki $e t$ al. [29] studied the wake structure behind a square cylinder for small and medium blockage coefficients (0.125-0.25) at Reynolds numbers ranging from 62 to 300. They evaluated the critical Reynolds number for the onset of vortex shedding and found that it increased as the blockage coefficient was raised. In addition, Zettner and Yoda [32] conducted experiments to observe effects due to confining walls. In the moderate range of blockage coefficient (0.3-0.6), their observation indicated that the angular separation points on the cylinder surface were much smaller than those in an unbounded flow.

On the other hand, an asymmetric blockage arises if the cylinder is placed away from the centerline of flow between two confining walls. Taneda [25] examined the effect of cylinder displacement away from the channel axis for blockage coefficients of $0.007,0.01$, and 0.04 and found a rapid increase as the cylinder was moved toward the wall. Later, Ramamurthy and Lee [21] carried out tests for a blockage coefficient of 0.31 and much larger Reynolds numbers in the regime of turbulent flow. More recently, Zovatto and Pedrizzetti [33] examined numerically the vortex shedding for the cylinder asymmetrically placed between the parallel walls. The blockage coefficient they set is 0.2 . They found that the vortex shedding occurred at a larger Reynolds number as the cylinder approached one wall and that the close proximity of one wall inhibited the appearance of vortex shedding.

It seems apparent that most studies of flow past a cylinder within confined walls attempted to capture the vortex shedding, its interaction with the shear flow arising from the existence of confining walls, and the vorticity dissipation mechanism. This is particularly true in the study with an asymmetric setting of the cylinder. It is the purpose in the present work to examine the effect of asymmetry of cylinder setting on the emergence and development of the recirculating flow. Of particular interest are the critical Reynolds number for its appearance and shape development at the early stage of appearance. The effect of shearing effects due to the confining walls will also be presented.

\section{MATHEMATICAL FORMULATION AND NUMERICAL DISCRETIZATION}

\section{Mathematical formulation}

Shown in Figure 1, a circular cylinder of diameter $d$ is asymmetrically placed in a channel bounded between two infinite parallel plates that extend upstream and downstream. The width between the two plates is $w$. The distance from the center of cylinder to the centerline of the channel is $a$. The fluid flow in the channel is governed by the Navier-Stokes equations that can be expressed in a non-dimensional form,

$$
\begin{aligned}
& \nabla \cdot \boldsymbol{U}=0 \\
& \boldsymbol{U} \cdot \nabla \boldsymbol{U}=-\nabla p+\frac{1}{\operatorname{Re}} \nabla^{2} \boldsymbol{U}
\end{aligned}
$$

where $\boldsymbol{U}(\boldsymbol{x})=(u(\boldsymbol{x}), v(\boldsymbol{x}))$ and $p(\boldsymbol{x})$ are the velocity and

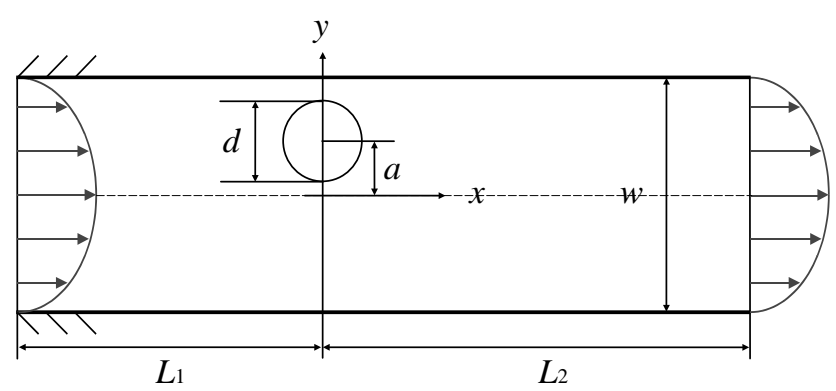

Fig. 1. Schema of the flow domain. 
pressure fields, respectively, at a field point $\boldsymbol{x}=(x, y)$, and $R e$ is the Reynolds number defined as

$$
\operatorname{Re} \equiv \frac{U_{\max } w}{v}
$$

in which $v$ is the kinematic viscosity of fluid and $U_{\max }$ is the maximum speed of the far upstream velocity profile.

To complete the mathematical formulation, we specify the boundary conditions as follows. A no-slip condition is prescribed on the plate and cylinder surfaces. In addition, a Poiseuille fully-developed velocity profile is prescribed at the far upstream and downstream boundaries

$$
\boldsymbol{U}(\boldsymbol{x})=U_{\max }\left[1-4\left(\frac{y}{w}\right)^{2}\right] \boldsymbol{i},
$$

far upstream and downstream.

This prescription corresponds to the flow condition under a constant streamwise pressure gradient without interference of the cylinder. The specification of far upstream and downstream boundary conditions has been justified by Amick [2] for sufficiently small Reynolds numbers. He has shown that for Reynolds numbers less than approximately 300 , disturbances induced by the presence of the cylinder decay exponentially upstream and downstream from the cylinder. Since the range of the Reynolds number in the present study is far less than 300 , it is legitimate to specify a fully-developed velocity profile as far upstream and downstream boundary conditions.

In addition to the dynamic parameter appearing in the governing equation, there are two geometric parameters in this flow setup. They are the blockage coefficient $B$ and the asymmetry coefficient $A$, defined, respectively, as

$$
B \equiv \frac{d}{w}, \text { and } A \equiv \frac{2 a}{w} .
$$

We vary these two parameters to study the effects of blockage and proximity of a confining wall on the development of recirculating eddy.

\section{Numerical discretization and domain truncation}

For computations, we employed a finite volume method with the SIMPLE scheme, proposed by Patankar [20]. This method uses a staggered grid for pressure and velocity fields. Since the computation procedure has been a standard one, the details are omitted here. For further discussions on discretization, see, e.g. [3].

Furthermore, the flow domain must be truncated in computations. As discussed above, Amick [2] has shown that disturbances induced by the cylinder decay exponentially both upstream and downstream from the cylinder. This property establishes a solid background for us to truncate the computation domain and prescribe a Poiseuille velocity distribution at some finite distances upstream and downstream of the cylinder. Furthermore, since the disturbance decay is exponential, it is eligible to compute solutions in a small domain without significantly distorting the flow development, as computationally justified by Chen [7]. In the present study, we set $L_{1}=3 d$ and $L_{2}=3 \mathrm{w}$, respectively.

\section{Grid generation}

We briefly address some features of the mesh used in the present study before discussing the results in details. The mesh in the present study was generated through the commercial package GRIDGEN.

It has been known that the structure of mesh plays an important role in computational fluid dynamics. In this study, we employed an $O$-type mesh around the cylinder, which was embedded in another $H$-type mesh. Two different meshes have been specifically designed according to the position of cylinder. Figure 2 shows these two types of typical mesh structures around the cylinder. The grid shown in Figure 2(a) is the one we used when the cylinder is far away from the lateral wall. However, when the cylinder is close to the wall, the mesh shown in Figure 2(b) was instead employed in order to maintain grid orthogonality and proper aspect ratios.

In the present study, the mesh numbers for different values of $B$ are between 110,000 and 260,000, depending on the required accuracy of computed solutions.

\section{RESULTS AND DISCUSSIONS}

The present study focuses on the emergences and developments of recirculating flow attached to the cylinder. To bring forth a clear picture of their developments, we carried out a series of careful computations for different values of $B$ and $A$. The former varies from 0.1 to 0.5 with an increment of 0.1 and the latter at a multiple of 0.1 from 0.1 .

\section{Tests of the present procedure}

The case for which $A=0$ represents a symmetric flow setting. In this situation, it has been known that a pair of recirculating region appears at the rear of the cylinder if the Reynolds number is not too small. We computed this flow and compared the results with those by Chen [7] which were obtained by a finite element method. These computations serve as the benchmark 
tests to determine a proper mesh size and meanwhile demonstrate the capability of the present procedure.

Two of the computed data are shown here. At $B=$ 0.1 , the critical Reynolds number for the emergence of cylinder-attached recirculating region was found to be 69.0 and 69.2 in the present study and Chen [7], respectively. Meanwhile, at $B=0.4$, they are 38.8 and 39.2 , respectively. It seems that the data in the present study tend to be somewhat smaller than those obtained by Chen [7]. However, they are comparable and show a reasonable agreement. The minor deviations may be due to different algorithms and numerical procedures.

\section{Single recirculating region}

Due to asymmetry of the flow geometry, we expect that the flow pattern behind the cylinder is no longer symmetric for a non-zero asymmetry coefficient. It is interesting to find that not only the symmetry but also the twin-vortices structure appearing in the symmetric

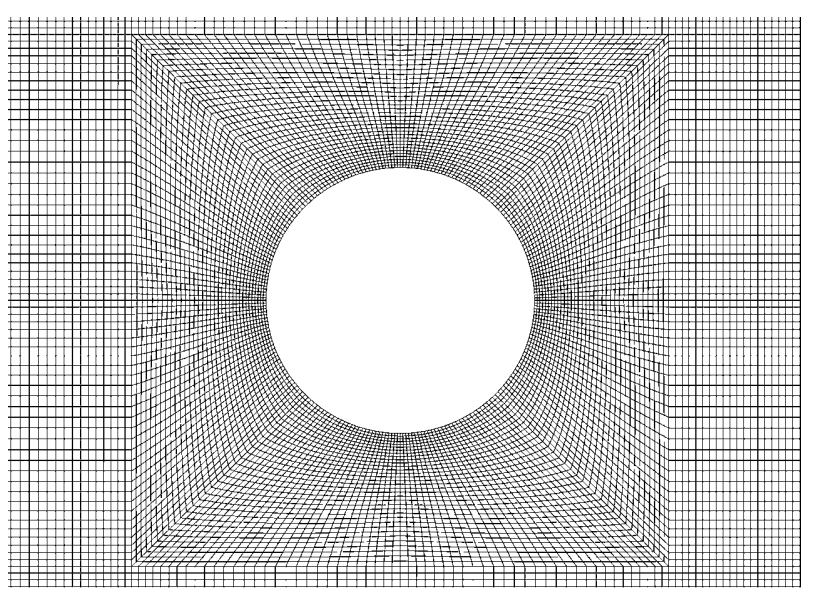

(a) Mesh for cylinder far away from the wall.

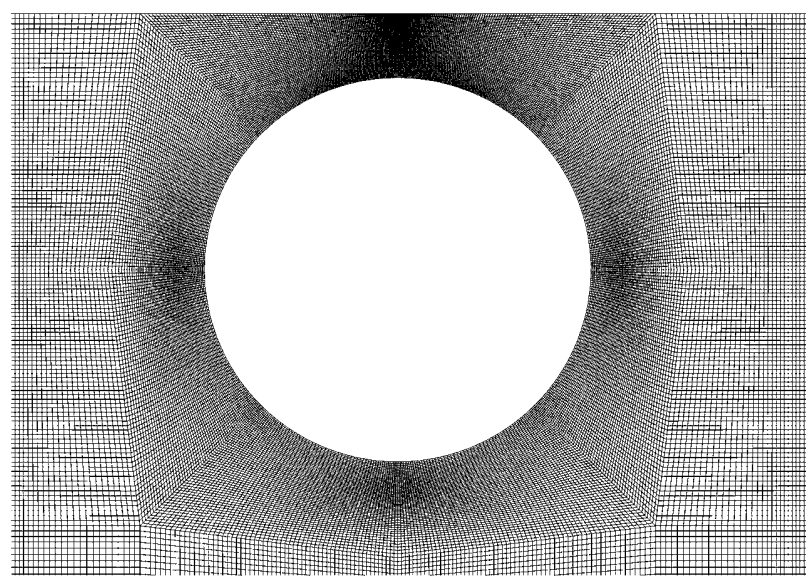

(b) Mesh for cylinder close to the wall.

Fig. 2. Typical meshes used in the present study. flow setting are destroyed. Instead, a single recirculating region attached to the cylinder appears.

Figures 3-5 shows some examples for some different values of asymmetric coefficients at $B=0.1,0.3$, and 0.5. They represent streamline patterns for small, medium, and large blockage coefficients, respectively. All streamlines shown in these plots were obtained by moving the cylinder upward as illustrated schematically in Figure 1. General observations show that the single recirculating region appears on the upper side of the cylinder where the fluid flow is slower. The fluid particles just outside the region move around it, reverse their directions as they pass the farthest point of the region, and finally reverse again as they move down-

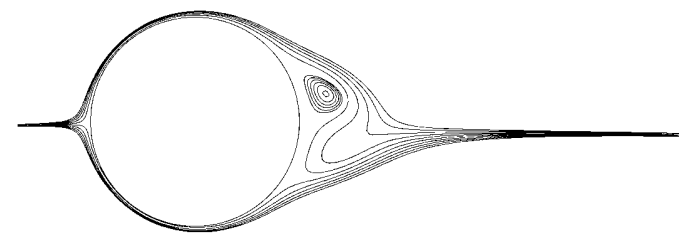

(a) $A=0.1(\operatorname{Re}=110)$.

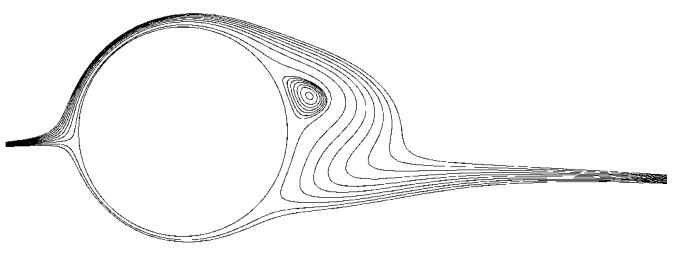

(b) $A=0.3(\operatorname{Re}=180)$.

Fig. 3. Single recirculating region for $B=0.1$.

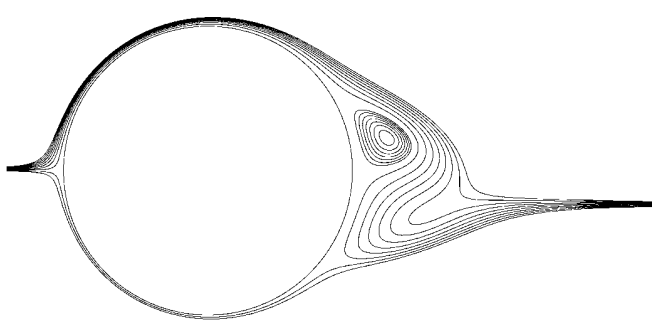

(a) $A=0.1(\operatorname{Re}=80)$.

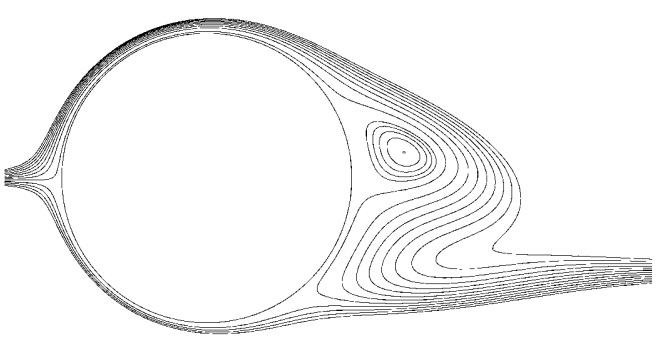

(b) $A=0.3(\operatorname{Re}=140)$.

Fig. 4. Single recirculating region for $B=0.3$. 
ward to join the faster stream from the lower side of the cylinder. The curvatures of the streamlines lying between the recirculating and the flow stream on the lower side are usually quite large. However, at this early stage of emergence of the recirculating flow, we did not find saddle points in this flow region.

The single recirculating region was also observed in a uniform flow past an elliptic cylinder inclined to the stream at some angles, the range of which depends on the Reynolds number and the minor-to-major axis ratio, as reported by Dennis and Young [12]. However, the mechanisms for it to appear seem different. The latter is due to the asymmetric setting of the elliptic cylinder on the incoming stream, while the former happens because of asymmetry of shear flow past the circular cylinder.

In addition, we also found in the computations that if the cylinder was placed too close (such as at $A=0.5$ and up for $B \leq 0.3, A=0.4$ and up for $B=0.4$ and $A=$ 0.3 and up for 0.5 ) to or attached (for all values of $B$ ) to one of the walls, it appears that there exists no cylinderattached recirculating region. In fact, a wall-attached one appears instead. This could be due to the fact that the proximity of the cylinder to the wall is equivalent, in effect, to an obstacle extruding from the wall. For the latter, a wall-attached recirculating region appears as the flow past it.

\section{Critical Reynolds numbers}

To find the critical Reynolds number for the onset of the single attached recirculating region, we employed an extrapolating procedure. First of all, we defined $L_{a}$ as the length of the recirculating region

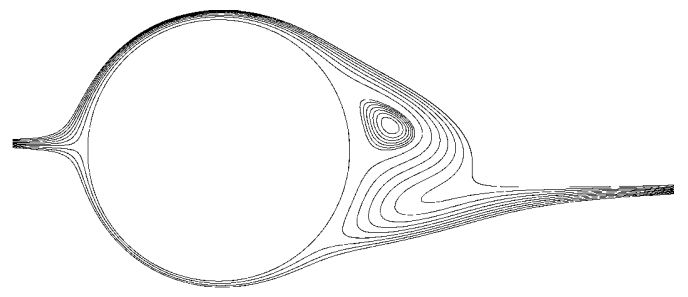

(a) $A=0.1(\operatorname{Re}=80)$.

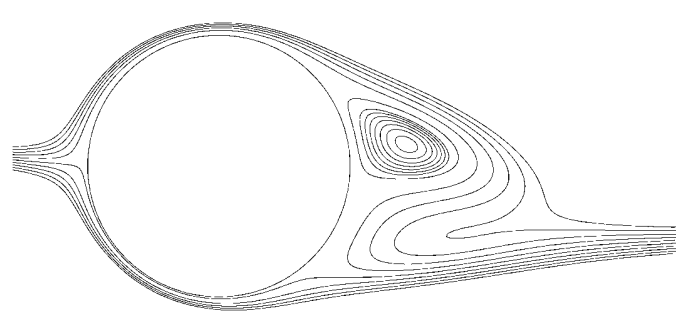

(b) $A=0.2(\operatorname{Re}=150)$.

Fig. 5. Single recirculating region for $B=0.5$. attached to the cylinder, which extends horizontally from the farthest point of the recirculating region to the point on the cylinder, as shown in Figure 6.

For selected asymmetry and blockage coefficients, we computed the flow for different Reynolds numbers, usually ranging from 50 to 200 . From the computed data, we located the farthest point on the recirculating eddy from the cylinder and, then, the length $L_{a}$ could be obtained through some arithmetic calculations. A relation between $L_{a}$ and Re was finally plotted and found through some proper curve fitting. And we then can extrapolate to find the critical Reynolds number for a particular set of blockage and asymmetry coefficients.

Figure 7 shows the elaborately computed results for various blockage and asymmetry coefficients. It is quite interesting to observe that in every case, the relation seems clearly linear. A regression analysis shows good linearity for each set of the computed data. It appears that at the early stage of development, the recirculating region grows linearly in length. The similar linear relationship has been reported theoretically by Simth [22] and computationally by Fornberg [13, 14] for an unbounded flow past a circular cylinder and by Chen et al. [9] for the flow past a circular cylinder symmetrically confined between two parallel plates. The length of the attached recirculating eddy seems to grow in a linear manner as the Reynolds number is increased at the early stage of its emergence, regardless of the boundary geometry around the cylinder.

The critical Reynolds number for the onset of the cylinder-attached vortex can then be obtained through a linear extrapolation. The results are presented in Table 1 and Figure 8.

These results obviously indicate that, for a fixed blockage coefficient, the cylinder-attached vortex rapidly delays to appear as the cylinder move toward the lateral wall. Nevertheless, this is somewhat expected because the fluid flow around the cylinder becomes slower as the cylinder is moved toward the wall. To examine in a more justifiable manner the wall effect on

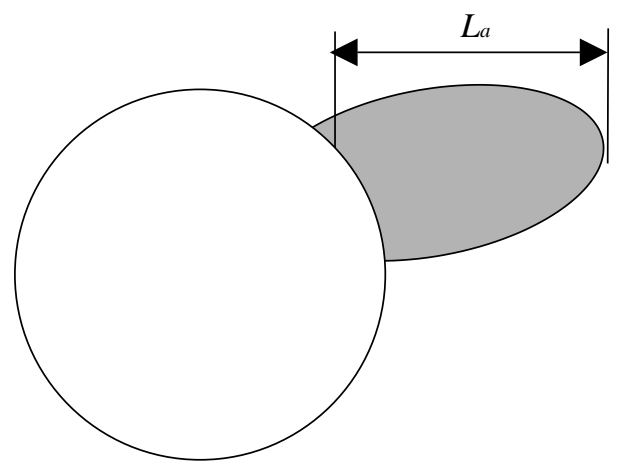

Fig. 6. Schematic definition for the length of single recirculating region. 

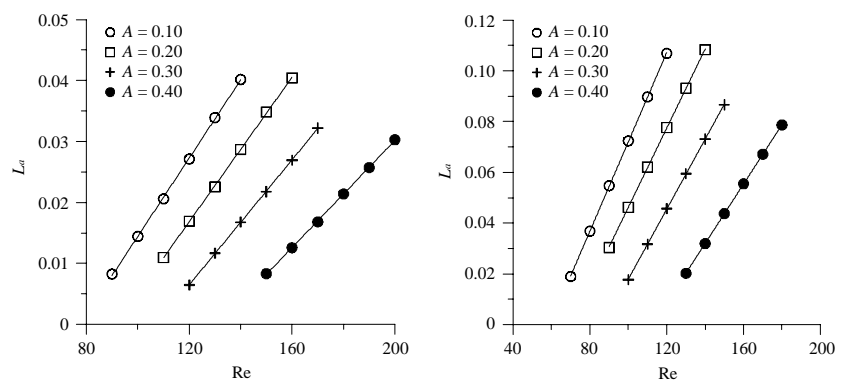

(a) $B=0.1$ (solid lines represent linear fitting).

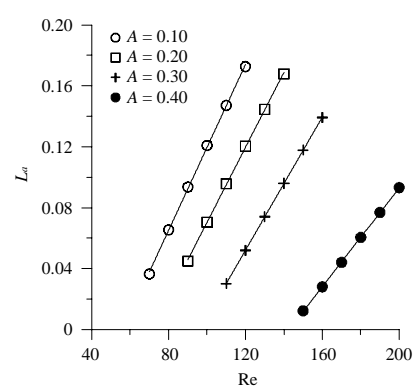

(c) $B=0.3$ (solid lines represent linear fitting).

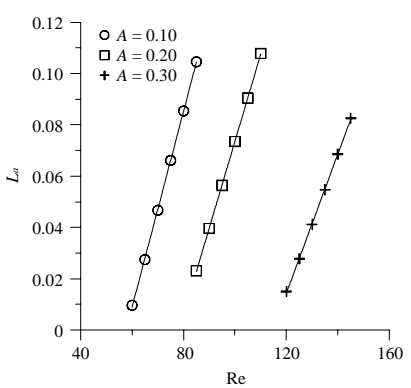

(d) $B=0.4$ (solid lines represent linear fitting).

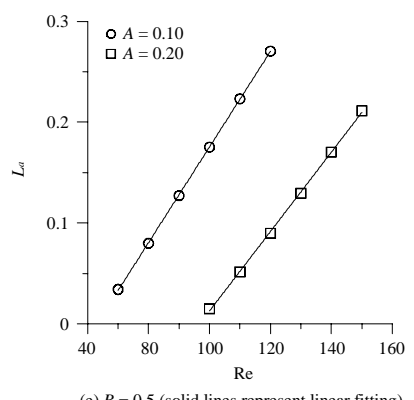

(e) $B=0.5$ (solid lines represent linear fitting)

Fig. 7. $L_{a}$-Re relationship for various blockage coefficients.

the emergence of attached recirculating region, we rescaled the Reynolds number based on the average speed $U_{\text {ave }}$ defined as

$$
U_{\text {ave }}=\frac{1}{d} \int_{y_{c}-d / 2}^{y_{c}+d / 2} u(\boldsymbol{x}) d y
$$

where $y_{c}$ is the $y$-coordinate at which the center of the cylinder is positioned and $u(\boldsymbol{x})$ the far-upstream velocity distribution in $x$-direction. The new Reynolds number is then defined as

$$
(\mathrm{Re})_{\text {ave }} \equiv \frac{U_{\text {ave }} d}{v}
$$

We recalculated the onset Reynolds numbers based on the new definition and the results are shown in Table 2 and Figure 9. These data still indicate that the onset value increases as the asymmetry coefficient become larger for a fixed blockage coefficient. Therefore, we may reasonably conclude that the presence of wall
Table 1. Onset Reynolds number for various blockage and asymmetry coefficients

\begin{tabular}{rrrrrc}
\hline & $B=0.1$ & $B=0.2$ & $B=0.3$ & $B=0.4$ & $B=0.5$ \\
\hline$A=0.1$ & 77.5 & 59.1 & 56.0 & 57.7 & 63.0 \\
$A=0.2$ & 91.5 & 70.4 & 71.4 & 78.3 & 96.8 \\
$A=0.3$ & 107.4 & 87.0 & 96.1 & 114.7 & NA \\
$A=0.4$ & 131.4 & 112.7 & 142.6 & NA & NA \\
\hline
\end{tabular}

Table 2. Onset Reynolds number based on Eq. (6)

\begin{tabular}{rrrrcc}
\hline & $B=0.1$ & $B=0.2$ & $B=0.3$ & $B=0.4$ & $B=0.5$ \\
\hline$A=0.1$ & 7.6 & 11.5 & 16.1 & 21.6 & 28.6 \\
$A=0.2$ & 8.8 & 13.3 & 19.9 & 28.4 & 42.4 \\
$A=0.3$ & 9.7 & 15.6 & 25.4 & 39.3 & NA \\
$A=0.4$ & 11.0 & 18.6 & 34.6 & NA & NA \\
\hline
\end{tabular}

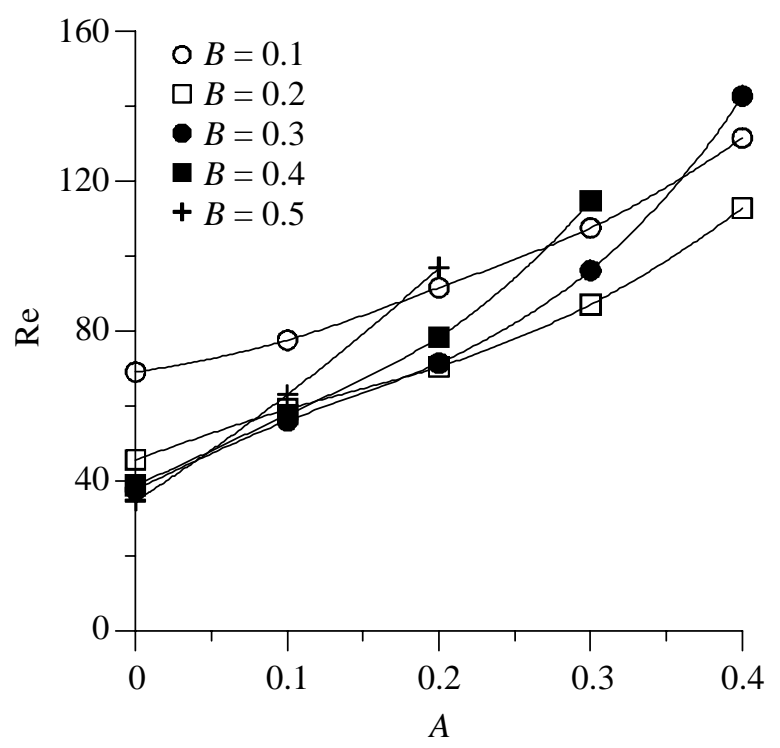

Fig. 8. Relation of the onset $\operatorname{Re}$ to $A$ at various values of $B$.

proximity does incur a suppressing effect on the emergence of the cylinder-attached recirculating region. The effect becomes more significant as the cylinder is moved more closely to the wall.

\section{Length growth rates}

Even though recirculating regions appear to grow linearly in length for all cases, they grow at different rates. We computed the length growth rates of recirculating region, $d L_{a} / d(\mathrm{Re})$, for different values of $B$ and $A$. The results are shown in Figure 10. They indicate that 


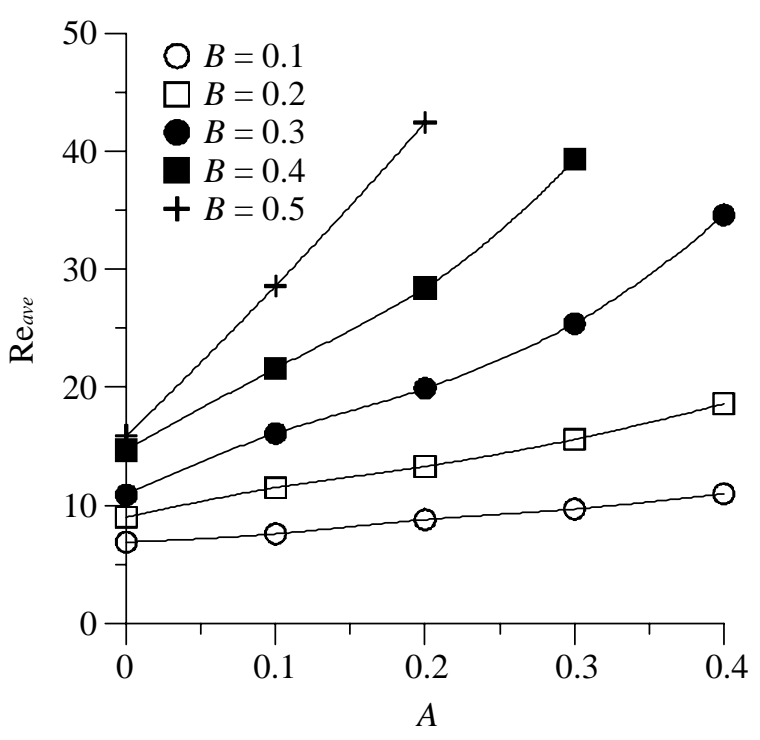

Fig. 9. Relation of the onset (Re) ave to $A$ at various values of $B$.

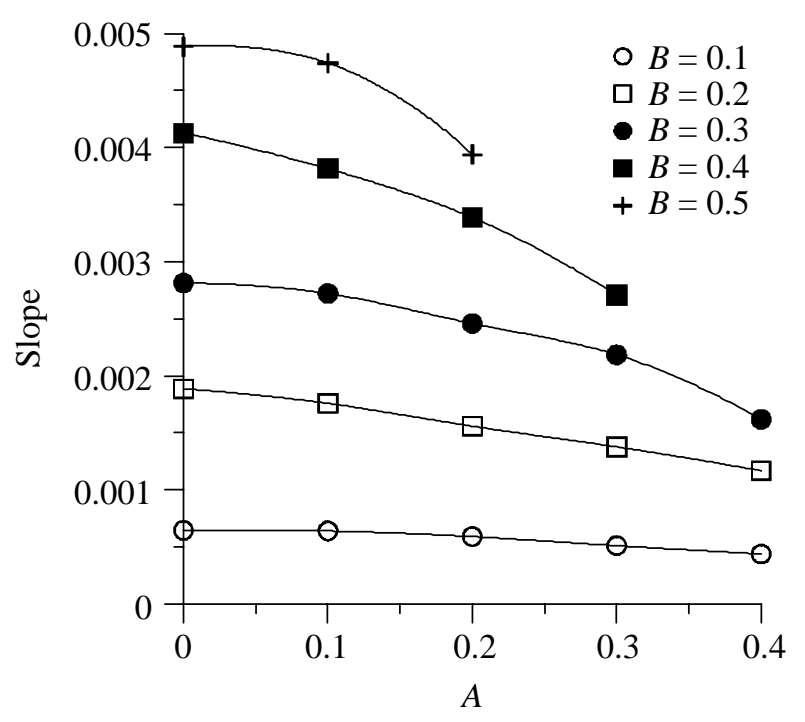

Fig. 10. The growth rates of the length of recirculating region.

for a fixed blockage coefficient, the length growth becomes somewhat slower at a larger asymmetry coefficient. This trend appears more significant for a larger blockage coefficient. This may arise owing, again, to the fact that the velocity distribution experienced by the cylinder becomes smaller for a larger asymmetry coefficient.

\section{CONCLUSION}

A study of a fully developed flow past a circular cylinder asymmetrically confined in a channel has been carried out through computations. Generally speaking, when the cylinder is moved toward the wall, the attached recirculating region becomes asymmetric and there exists only one vortex inside it. The cylinderattached recirculating region grows linearly as the Reynolds number is increased. This fact makes it easy to find the critical Reynolds number for the onset of the recirculating region by linear extrapolations.

When the cylinder is too close to the lateral wall, a wall-attached recirculating region is formed. Under this situation, the single cylinder-attached recirculating region does not appear at the range of Reynolds numbers in our present study. The existence of lateral walls suppresses the formation of cylinder-attached recirculating region and its effect becomes more significant for a large blockage coefficient.

Finally, we also examined the growth rate of the recirculating region. It is evident that it depends on the blockage and asymmetry coefficients. In addition, as the cylinder is moved towards one of the walls, it grows more slowly.

\section{ACKNOWLEDGEMENTS}

This work was supported by the National Science Council, Republic of China, under grant NSC 93-2611E-019-021. The authors would like to express their thanks for this support.

\section{REFERENCES}

1. Acrivos, A., Leal, L.G., Snowden, D.D., and Pan, F., "Further Experiments on Steady Separated Flow Past Bluff Objects," Journal of Fluid Mechanics, Vol. 34, pp. 25-48 (1968).

2. Amick, C.J., "Properties of Steady Navier-Stokes Solutions for Certain Unbounded Channels and Pipes," Nonlinear Analysis, Theory, Methods, and Applications, Vol. 2, pp. 689-720 (1978).

3. Anderson, J.D., Computational Fluid Dynamics, McGraw-Hill, Singapore (1995).

4. Anderson, C.R. and Reider, M.B., "A High Order Explicit Method for the Computation of Flow About a Circular Cylinder," Journal of Computational Physics, Vol. 125, pp. 207-224 (1996).

5. Bearman, P.W. and Zdravkovich, M.M., "Flow Around a Circular Cylinder Near a Plane Boundary," Journal of Fluid Mechanics, Vol. 89, pp. 33-47 (1978).

6. Behr, M., Hastreiter, D., Mittal, S., and Tezduyar, T.E., "Incompressible Flow Past a Circular Cylinder: Dependence of the Computed Flow Field on the Location of the Lateral Boundaries," Computer Methods in Applied Mechanics and Engineering, Vol. 123, pp. 309-316 (1995). 
7. Chen, J.-H., "Wall Effects on Separation of Flow Past a Circular Cylinder," Journal of the Chinese Institute of Engineers, Vol. 16, No. 3, pp. 333-344 (1993).

8. Chen, J.-H., "Laminar Separation of Flow Past a Circular Cylinder between Two Parallel Plates," Proceedings of National Science Council, ROC, Series A, Vol. 24, No. 5, pp. 341-351 (2000).

9. Chen, J.-H., Pritchard, W.G., and Tavener, S.J., "Bifurcation for Flow Past a Cylinder between Parallel Planes," Journal of Fluid Mechanics, Vol. 284, pp. 2341 (1995).

10. Coutanceau, M. and Bouard, R., "Experimental Determination of the Main Features of the Viscous Flow in the Wake of a Circular Cylinder in Uniform Translation. Part 1. Steady Flow," Journal of Fluid Mechanics, Vol. 79, pp. 231-256 (1977).

11. Coutanceau, M. and Bouard, R., "Experimental Determination of the Main Features of the Viscous Flow in the Wake of a Circular Cylinder in Uniform Translation. Part 2. Unsteady Flow," Journal of Fluid Mechanics, Vol. 79, pp. 257-272 (1977).

12. Dennis, S.C.R. and Young, P.J.S., "Steady Flow Past an Elliptic Cylinder Inclined to the Stream," Journal of Engineering Mathematics, Vol. 47, pp. 101-120 (2003).

13. Fornberg, B., "A Numerical Study of Steady Viscous Flow Past a Circular Cylinder," Journal of Fluid Mechanics, Vol. 98, pp. 819-855 (1980).

14. Fornberg, B., "Steady Viscous Flow Past a Circular Cylinder Up to Reynolds Number 600," Journal of Computational Physics, Vol. 61, pp. 297-320 (1985).

15. Gerrard, J.H., "The Wakes of Cylindrical Bluff Bodies at Low Reynolds Number," Philosophical Transactions of the Royal Society of London, Vol. A 288, pp. 351-382 (1978).

16. Grove, A.S., Shair, F.M., Petersen, E.E., and Acrivos, A., "An Experimental Investigation of the Steady Separated Flow Past a Circular Cylinder," Journal of Fluid Mechanics, Vol. 19, pp. 60-80 (1965).

17. Lai, M.-C. and Peskin, C.S., "An Immersed Boundary Method with Formal Second-Order Accuracy and Reduced Numerical Viscosity," Journal of Computational Physics, Vol. 160, pp. 705-719 (2000).

18. Lei, C., Cheng, L., and Kavanagh, K., "Re-examination of the Effects of a Plane Boundary on Force and Vortex Shedding of a Circular Cylinder," Journal of Wind Engineering and Industrial Aerodynamics, Vol. 80, pp. 263-286 (1999).

19. Nishioka, M. and Sato, H., "Measurements of Velocity Distributions in the Wake of a Circular Cylinder at Low Reynolds Numbers," Journal of Fluid Mechanics, Vol. 65, pp. 97-112 (1974).

20. Patankar, S.V., Numerical Heat Transfer and Fluid
Flow, Hemisphere Publishing Corporation, Washington, D.C. (1980).

21. Ramamurthy, A.S. and Lee, P.M., "Wall Effects on Flow Past Bluff Bodies," Journal of Sound and Vibration, Vol. 31, pp. 433-451 (1973).

22. Smith, F.T., "Laminar Flow of an Incompressible Fluid Past a Bluff Body: the Separation, Reattachment, Eddy Properties and Drag," Journal of Fluid Mechanics, Vol. 92, pp. 171-205 (1979).

23. Sohankar, A., Norberg, C., and Davidson, L., "LowReynolds-Number Flow Around a Square Cylinder at Incidence: Study of Blockage, Onset of Vortex Shedding and Outlet Boundary Condition," International Journal for Numerical Methods in Fluids, Vol. 26, pp. 39-56 (1998).

24. Taneda, S., "Experimental Investigation of the Wakes Behind Cylinders and Plates at Low Reynolds Numbers," Journal of the Physical Society of Japan, Vol. 11, pp. 302-307 (1956).

25. Taneda, S., "Experimental Investigation of the WallEffect on a Cylindrical Obstacle Moving in a Viscous Fluid at Low Reynolds Numbers," Journal of the Physical Society of Japan, Vol. 19, pp. 1024-1030 (1964).

26. Tang, H.S., Jones, S.C., and Sotiropoulos, F., "An Overset-Grid Method for 3-D Unsteady Incompressible Flows," Journal of Computational Physics, Vol. 191, pp. 567-600 (2003).

27. Taniguchi, S. and Miyakoshi, K., "Fluctuating Fluid Forces Acting on a Circular Cylinder and Interference with a Plane Wall," Experiments in Fluids, Vol. 9, pp. 197-204 (1990).

28. Tritton, D.J., "Experiments on the Flow Past a Circular Cylinder at Low Reynolds Numbers," Journal of Fluid Mechanics, Vol. 6, pp. 547-567 (1956).

29. Turki, S., Abbassui, H., and Nasrallah, S.B., "Effect of Blockage Ratio on the Flow in a Channel with a Built-in Square Cylinder," Computational Mechanics, Vol. 33, pp. 22-29 (2003).

30. Zdravkovich, M.M., Flow Around Circular Cylinder, Vol. 1: Fundamentals. Oxford University Press, Oxford (1997).

31. Zdravkovich, M.M., Flow Around Circular Cylinder, Vol. 2: Applications. Oxford University Press, Oxford (2002).

32. Zettner, C.M. and Yoda, M., "The Circular Cylinder in Simple Shear at Moderate Reynolds Numbers: An Experimental Study," Experiments in Fluids, Vol. 30, pp. 346-353 (2001).

33. Zovatto, L. and Pedrizzetti, G., "Flow about a Circular Cylinder between Parallel Walls," Journal of Fluid Mechanics, Vol. 440, pp. 1-25 (2001). 